\title{
ESTUDO DA MORBIDADE NA UNIDADE DE INTERNAÇÃO DE ORTOPEDIA ${ }^{1}$
}

\author{
MORBIDITY STUDY OF THE ORTHOPEDIC UNIT
}

Marcia RAC Melo; Clarice A Ferraz²; Carla R Souza ${ }^{3}$ \& Marco AS Lavrador ${ }^{4}$

\begin{abstract}
${ }^{1,2}$ Docente do Departamento de Enfermagem Geral e Especializada - Escola de Enfermagem de Ribeirão Preto-USP. ${ }^{2}$ Coordenadora do Projeto Integrado de Pesquisa: Gestão da Qualidade e Produtividade na Enfermagem - CNPq (1997/1999). ${ }^{3}$ Enfermeira, Bolsista do Programa de Aperfeiçoamento de Ensino, ano de 1998. ${ }^{4}$ Docente do Departamento de Física e Química - Faculdade de Ciências Farmacêuticas de Ribeirão Preto - USP.

CorRespondêncIA: Marcia Regina A. Costa Melo - Avenida Bandeirantes, 3900 - 14040-902 - Ribeirão Preto -SP. e-mail: mracmelo@glete.eerp.usp.br
\end{abstract}

MELO MRAC; FERRAZ CA; SOUZA CR \& LAVRADOR MAS. Estudo da morbidade na unidade de internação de ortopedia. Medicina, Ribeirão Preto, 33: 73-81, jan./mar. 2000.

RESUMO: Modelo de estudo: Estudo retrospectivo e descritivo. Objetivo: Descrever a clientela hospitalizada na Unidade de Internação de Ortopedia do HCRP, segundo as variáveis do diagnóstico principal, idade e sexo. Metodologia: Foram analisadas as saídas hospitalares codificadas pela Classificação Internacional de Doenças, 9a revisão, 1975, ocorridas no período de 1992 a 1996. Os dados foram obtidos através do Serviço de Arquivo Médico (SAME) da instituição, a partir de programa de registro de altas da Companhia de Processamento de Dados do Estado de São Paulo- PRODESP e decodificados a partir da elaboração especial de um programa de computador desenvolvido em linguagem $\mathrm{C}++$. Resultados: Encontram-se 5819 saídas hospitalares, sendo o Capítulo XVII - Lesões e Envenenamentos - responsável pelo maior número, correspondendo a 2.345 saídas (40,30\%). Em seguida, o Capítulo XIII - Doenças do Sistema Osteomuscular e do Tecido Conjuntivo - foi o responsável por 1.904 saídas (32,72\%). O Capítulo VI - Doenças do Sistema Nervoso e dos Órgãos dos Sentidos - foi responsável por 433 saídas (7,44\%). Especificamente, o diagnóstico de Síndrome do Túnel do Carpo representou 310 casos. O Capítulo II - Neoplasma - foi o responsável por 479 saídas $(8,23)$. O Capítulo XIV - Anomalias Congênitas foi o responsável por 333 saídas (5,72\%). Os demais capítulos apresentaram freqüência menor de saídas. Em todos os capítulos notou-se distribuição nas diversas faixas etárias, predominando a de 20 a 49 anos de idade (48,44\%). Considerações: Acredita-se que o conhecimento da morbidade hospitalar, pelos enfermeiros, possa orientar o uso de recursos dos serviços de saúde, de modo a garantir a qualidade da assistência prestada.

UNITERMOS: Morbidade. Enfermagem Ortopédica. Unidades Hospitalares. Ortopedia.

\section{INTRODUÇÃO}

A implementação das diretrizes do Sistema Único de Saúde (SUS), dentre elas a hierarquização e a eqüidade no acesso, tem provocado mudanças na organização do atendimento hospitalar. A adesão do Hos- pital das Clínicas de Ribeirão Preto (HCRP) ao SUS, desde 1988, permitiu aos profissionais de enfermagem que ali trabalham a percepção de que a clientela atendida está diferente: portadores de patologias mais complexas ou de doenças crônicas em estágio mais avançado, com restabelecimento mais difícil e maior

Trabalho apresentado no II Encontro Nacional de Gerenciamento em Enfermagem para o Século XXI - "Gerenciando o desenvolvimento de talentos humanos na enfermagem", 12 a 14 de agosto de 1999. 
grau de dependência da enfermagem; além de que as internações têm sido em períodos menores, sucedidos de reinternações ${ }^{(1)}$.

A mudança com a clientela exigiu o aumento das especialidades médicas e a implementação de tecnologia de ponta. Desta forma, torna-se fundamental conhecer as mudanças ocorridas no perfil de morbidade hospitalar, a fim de implementar estratégias que possam implementar/qualificar o cuidado prestado nessa instituição, ápice da hierarquização do sistema de saúde.

Há de se considerar, também, o processo de transição epidemiológica no Brasil. Na década de 80, a queda das doenças infecciosas como causa de morte se manteve e o predomínio das doenças crônicas-degenerativas e dos traumas e violências se acentuou, ocorrendo um aumento das taxas de mortalidade por homicídios e traumas, causados por veículos automotores e pelas neoplasias ${ }^{(2)}$. Entre 1930 e 1986, a mortalidade por causas externas triplicou no Brasil, consequiência do processo de urbanização maciça e da proliferação dos bolsões de miséria das grandes cidades, sendo os acidentes de trânsito o segmento que mais contribuiu para essa incidência ${ }^{(3)}$. Além da mortalidade, é preciso considerar a importância dos acidentes, por serem causadores de incapacidade, invalidez permanente e mutilação ${ }^{(4)}$.

O conhecimento da estatística hospitalar é importante para a apreciação da clientela atendida. $\mathrm{O}$ seu uso, freqüentemente, está voltado para os aspectos administrativos, como previsão orçamentária, planejamento de recursos materiais e humanos, e, também, para os aspectos clínico e epidemiológico, subsidiando a avaliação da qualidade da assistência prestada pela instituiçãão ${ }^{(5)}$.

Especificamente, em relação à especialidade de Ortopedia, houve aumento do número de pacientes encaminhados para atendimento, tendo em vista ser o Hospital das Clínicas de Ribeirão Preto um hospital de referência terciária para cidades pertencentes à região, e possuir equipe capacitada e tecnologia de ponta para $\mathrm{o}$ atendimento, principalmente no que se refere à Traumatologia. Esse quadro repercute diretamente na Unidade de Internação de Ortopedia, merecendo estudo específico para aprofundar conhecimentos sobre as transformações ocorridas no perfil epidemiológico deste setor hospitalar, o qual procuramos identificar neste estudo.

\section{OBJETIVO}

Descrever a clientela hospitalizada na Unidade de Internação de Ortopedia do Hospital das Clínicas de Ribeirão Preto (HCRP), segundo as variáveis do diagnóstico principal, idade e sexo, no período de 1992 a 1996.

\section{MATERIAL E MÉTODO}

O estudo é de natureza descritiva, tipo "survey", que, segundo Polit \& Hungler ${ }^{(6)}$, visa obter informações quanto à prevalência, distribuição e interrelações de variáveis de uma população. Trivinõ s ${ }^{(7)}$ refere que o estudo descritivo pretende descrever "com exatidão" os fatos e fenômenos de determinada realidade.

O HCRP é um hospital geral, nível terciário na hierarquia do sistema de saúde, que atende a tríplice finalidade (ensino, pesquisa e assistência) e fornece assistência médica integral nas diversas especialidades, tanto em nível ambulatorial quanto em internações hospitalares. A Unidade de Ortopedia atende clientes nas diversas subespecialidades, incluindo adultos e crianças.

Após o aval da Comissão de Ética do HCRP, os dados foram obtidos no Serviço de Arquivo Médico (SAME) através do Programa de Registros de Altas da Companhia de Processamento de Dados do Estado de São Paulo - PRODESP, no período de 1992 a 1996 (5819 saídas).

Tais dados foram decodificados a partir da elaboração especial de um programa de computador, desenvolvido em linguagem $\mathrm{C}++$, e analisados segundo variáveis: diagnóstico principal, registrado na saída hospitalar, de acordo com a Classificação Internacional de Doenças (CID), $9^{a}$ Revisão, $1995^{(8)}$; sexo e idade que foi agrupada em oito faixas de modo a contemplar as diferentes fases do desenvolvimento humano.

\section{RESULTADOS E DISCUSSÃO}

A Unidade de Internação de Ortopedia do HCRP possuía, no período estudado, 44 leitos distribuídos em dez enfermarias. A Unidade foi responsável por 5.819 internações no período de 1992 a 1996. Em relação ao diagnóstico principal, os resultados encontram-se na Tabela I, sendo que se optou por destacar os diagnósticos prevalentes.

Observa-se, na Tabela I, que os Neoplasmas (CID 140 - 239) corresponderam a 479 saídas (8,23\%). Destacamos, naquele capítulo, o neoplasma maligno de ossos e cartilagens articulares com 135 casos $(2,32 \%)$ e o neoplasma de natureza não especificada com 102 casos $(1,75 \%)$. 

Tabela I - Distribuicão numérica e percentual das saídas hospitalares na Unidade de Ortopedia do HCRP.
segundo o diagnóstico principal, no período de 1992 a 1996, Ribeirão Preto, 1999

\begin{tabular}{|llrc|}
\hline CID & Diagnóstico principal & No & $\%$ \\
\hline Capítulo I - Doenças infecciosas e parasitárias (001-139) & & \\
\hline 030 & Hanseníase & 11 & 0,19 \\
138 & Efeitos tardios da poliomielite aquda & 14 & 0,24 \\
- & Demais & 17 & 0,29 \\
\hline Capítulo II - Neoplasmas (140-239) & & \\
\hline 170 & Neoplasma maligno dos ossos e das cartilagens articulares & 135 & 2,32 \\
171 & Neoplasma maligno do tecido coniuntivo e de outros tecidos moles & 40 & 0,69 \\
173 & Outros neoplasmas malignos da pele & 21 & 0,36 \\
198 & Neoplasma maligno secundário de outras localizaç̃̃es especificadas & 31 & 0,53 \\
213 & Neoplasma beniqno dos ossos e das cartilaqens articulares & 18 & 0,31 \\
215 & Outros neoplasmas benignos do tecido coniuntivo e de outros tecidos moles & 33 & 0,57 \\
239 & Neoplasmas de natureza não especificada & 102 & 1,75 \\
& Demais & 99 & 1,70
\end{tabular}

Capítulo III - Doenças das glândulas endócrinas, nutrição, metabolismo e transtornos imunitários(240-279)

Deficiência de vitamina $D$, gota dentre outros

$19 \quad 0,33$

Capítulo VI - Doenças do sistema nervoso e dos órgãos dos sentidos (320-389)

\begin{tabular}{|c|c|c|c|}
\hline 336 & Outras doenças da medula espinhal & 39 & 0,67 \\
\hline 343 & Paralisia cerebral infantil & 27 & 0,46 \\
\hline 354 & Mononeurite do membro superior e múltiplas & 310 & 5,33 \\
\hline \multirow[t]{2}{*}{355} & Mononeurite do membro inferior & 25 & 0,43 \\
\hline & Demais & 32 & 0,55 \\
\hline \multicolumn{4}{|c|}{ Capítulo XII - Doenças da pele e do tecido celular subcutâneo(680-709) } \\
\hline \multirow[t]{2}{*}{707} & Úlcera crônica de pele & 16 & 0,28 \\
\hline & Demais & 33 & 0,57 \\
\hline
\end{tabular}

Capítulo XIII - Doenças do sistema osteomuscular e do tecido conjuntivo(710-739)

\begin{tabular}{|llrc|}
\hline 111 & Artropatias associadas à infecção & 43 & 0,74 \\
715 & Osteoartroses e transtornos afins & 210 & 3,60 \\
717 & Desarranios internos do ioelho & 159 & 2,73 \\
718 & Outros desarranios das articulações & 65 & 1,12 \\
722 & Transtornos dos discos intervertebrais & 89 & 1,53 \\
726 & Entesopatias periféricas e síndromes afins & 113 & 1,94 \\
727 & Outros transtornos das sinóvias, tendões e da bolsa sinovial & 156 & 2,68 \\
730 & Osteomielite, periostite e outras infecções que atingem os ossos & 108 & 1,86 \\
732 & Osteocondropatias & 167 & 2,87 \\
733 & Outros transtornos dos ossos e das cartilagens (osteoporose, fratura patológica e e & \\
& outros) & 364 & 6,26 \\
736 & Outras deformidades adquiridas dos membros & 116 & 1,99 \\
737 & Desvio da coluna vertebral & 115 & 1,98 \\
& Demais & 199 & 3,42 \\
\hline
\end{tabular}




\begin{tabular}{|c|c|c|c|}
\hline CID & Diagnóstico principal & № & $\%$ \\
\hline \multicolumn{4}{|c|}{ Capítulo XIV - Anomalias congênitas(740-759) } \\
\hline 741 & Espinha bífida & 11 & 0,19 \\
\hline 754 & Algumas deformidades osteomusculares congênitas & 162 & 2,79 \\
\hline 755 & Outras anomalias congênitas dos membros & 78 & 1,34 \\
\hline \multirow[t]{2}{*}{756} & Outras anomalias osteomusculares congênitas & 74 & 1,27 \\
\hline & Demais & 8 & 0,14 \\
\hline \multicolumn{4}{|c|}{ Capítulo XV - Algumas afecções originadas no período perinatal(760-769) } \\
\hline 767 & Traumatismo ocorrido durante o nascimento & 14 & 0,24 \\
\hline \multicolumn{4}{|c|}{ Capítulo XVI - Sintomas, sinais e afecções definidas(780-799) } \\
\hline \multirow[t]{2}{*}{782} & Sintomas relativos a pele e a outros tecidos do tegumento & 17 & 0,29 \\
\hline & Demais & 21 & 0,36 \\
\hline \multicolumn{4}{|c|}{ Capítulo XVII - Lesões e envenenamentos(800-999) } \\
\hline 805 & Fratura da coluna vertebral sem menção de lesão da medula espinhal & 167 & 2,87 \\
\hline 806 & Fratura da coluna vertebral com menção de lesão da medula espinhal & 25 & 0,43 \\
\hline 808 & Fratura da bacia & 44 & 0,75 \\
\hline 812 & Fratura do úmero & 89 & 1,53 \\
\hline 813 & Fratura do rádio e do cúbito & 105 & 1,81 \\
\hline 815 & Fratura de ossos do metacarpo & 49 & 0,84 \\
\hline 816 & Fratura de uma ou de várias falanges da mão & 49 & 0,84 \\
\hline 820 & Fratura do colo do fêmur & 160 & 2,75 \\
\hline 821 & Fratura de outras partes, e de partes não especificadas do fêmur & 155 & 2,67 \\
\hline 823 & Fratura da tíbia e do perônio & 96 & 1,65 \\
\hline 824 & Fratura do tornozelo & 38 & 0,65 \\
\hline 881 & Ferimentos do cotovelo, antebraço e do punho & 43 & 0,74 \\
\hline 883 & Ferimentos de um ou de vários dedos da mão & 91 & 1,56 \\
\hline 905 & Efeitos tardios de lesões traumáticas osteomusculares e do tecido conjuntivo & 403 & 6,93 \\
\hline 906 & Efeitos tardios de lesões traumáticas da pele e dos tecidos subcutâneos & 53 & 0,91 \\
\hline 907 & Efeitos tardios de lesões traumáticas do sistema nervoso & 71 & 1,22 \\
\hline 955 & Traumatismos de nervo periférico da cintura escapular e membro superior & 77 & 1,32 \\
\hline \multirow[t]{2}{*}{996} & Complicações peculiares a alguns procedimentos especificados & 233 & 4,01 \\
\hline & Demais & 397 & 6,82 \\
\hline \multicolumn{2}{|c|}{ Outros capítulos (IV, VII, VIII E X) } & 17 & 0,29 \\
\hline \multicolumn{4}{|c|}{$\begin{array}{l}\text { Classificacão suplementar de fatores que exercem influência sobre o estado de saúde e de oportunidades de } \\
\text { contato com serviços de saúde (V01-V82) }\end{array}$} \\
\hline V45 & Outros estados pós-cirúrgicos & 23 & 0,40 \\
\hline \multirow[t]{2}{*}{ V54 } & Outros cuidados de segmento em Ortopedia & 113 & 1,94 \\
\hline & Demais & 7 & 0,12 \\
\hline \multicolumn{2}{|c|}{ Não válidos } & 3 & 0,05 \\
\hline \multicolumn{2}{|c|}{ Total } & 5.819 & 100,00 \\
\hline
\end{tabular}


Em relação às Doenças do Sistema Nervoso e dos Sentidos (CID 320 - 389), elas corresponderam a 433 saídas $(7,44 \%)$. Especificamente, a mononeurite do membro superior e mononeurites múltiplas representaram 310 casos (5.33\%), incluindo a síndrome do túnel do carpo e as lesões do nervo ulnar e radial.

As Doenças do Sistema Osteomuscular e do Tecido Conjuntivo (CID 710 - 739) foram responsáveis por 1.904 saídas $(32,72 \%)$. Destacamos no capítulo: Outros Transtornos dos Ossos ou Cartilagens com 364 casos $(6,26 \%)$, incluindo a osteoporose, a fratura patológica, o cisto ósseo, a ausência ou consolidação de fratura, o pé plano; Osteoartrose e Transtornos Afins com 210 casos $(3,60 \%)$; Osteocondropatias com 167 casos (2,87\%); Desarranjos Internos do Joelho com 159 casos (2,73\%); Outros Transtornos de Sinóvias, dos Tendões e da Bolsa Sinovial com 156 casos $(2,68 \%)$, incluindo sinovite e tenossinovite, joanete, gânglios e cistos da sinóvia, do tendão e da bolsa sinovial; Outras Deformidades Adquiridas dos Membros com 116 casos (1,99\%); Desvio da Coluna Vertebral com 115 casos (1,98\%); Entesopatias Periféricas e Síndromes Afins com 113 casos (1,94\%), incluindo a síndrome do manguito rotator do ombro e, osteomielite, periostite e outras infecções que atingem os ossos com 108 casos $(1,86 \%)$.

As Anomalias Congênitas (CID 740 - 759) corresponderam a 333 saídas $(5,72 \%)$. Dentre elas, algumas deformidades osteomusculares congênitas ocorreram em 162 casos $(2,79 \%)$ incluindo as deformidades da coluna vertebral, a luxação congênita de quadril, o "genu recurvatum" congênito, as deformidades dos pés.

Constituindo-se no maior grupo dentro da Unidade de Internação de Ortopedia, as Lesões e Envenenamentos (CID 800 - 999) foram responsáveis por 2.345 saídas $(40,30 \%)$, dados que corroboram com vários estudos, os quais referem que o maior número de vítimas encontra-se entre os adultos jovens, do sexo masculino, em decorrência de acidentes de trabalho, de trânsito, ou, ainda, por ser o grupo mais exposto à violência interpessoal ${ }^{(9 / 14)}$.

Destacam-se, dentre as Lesões e Envenenamentos, os efeitos tardios de lesões traumáticas osteomusculares e do tecido conjuntivo com 403 casos $(6,93 \%)$ incluindo os efeitos tardios das fraturas, luxações e lesões traumáticas de tendão; as complicações peculiares a alguns procedimentos especificados com $233 \mathrm{ca}$ sos $(4,01 \%)$ incluindo as complicações mecânicas de dispositivo, implante e enxerto, as infecções ou reações inflamatórias devidas à prótese interna, implante ou enxerto; as fraturas de coluna vertebral sem men- ção de lesão de medula espinhal com 167 casos (2,87\%); as fraturas de colo de fêmur com 160 casos $(2,75 \%)$; as fraturas de outras partes, e partes não especificadas do fêmur com 155 casos $(2,67 \%)$; as fraturas de rádio e cúbito com 105 casos $(1,81 \%)$ e as fraturas de tíbia e perôneo com 96 casos $(1,65 \%)$. Chama a atenção que, das 2345 saídas hospitalares devidas ao capítulo Lesões e Envenenamento, 526 corresponderam a fraturas dos membros inferiores e 426 dos membros superiores, coincidentes com outros achados ${ }^{(5,15)}$.

As Doenças Infecciosas e Parasitárias (CID 001$139)$ foram responsáveis por $42(0,72 \%)$ saídas; as Doenças de Glândulas Endócrinas, Nutrição, Metabolismo e Transtornos Imunitários (CID 240-269) por 19 saídas (0,32\%); as Doenças da Pele e do Tecido Celular Subcutâneo (CID 680-70) por 49 saídas $(0,85 \%)$; algumas Afecções, originadas no Período Perinatal (CID 760-769), 14 saídas (0,24\%); Sintomas, Sinais e Afecções Definidas (CID 780-799) 38 saídas (0,75\%) e Outros Capítulos (IV, VII, VIII e X) 17 saídas (0,29\%).

A seguir, na Tabela II, está descrita a incidência dos capítulos do CID.

Observa-se, na Tabela II, a maior predominância do capítulo XIII, pela frequiência de patologias ortopédicas, e do capítulo XVII, pela traumatologia, corroborando com os $20 \%$ de todos os leitos hospitalares, ocupados por pacientes traumatizados nos Estados Unidos da América ${ }^{(15)}$.

Em relação ao sexo, das 5.819 saídas, 3.254 $(55,9 \%)$ foram do sexo masculino e $2.562(44,1 \%)$ do sexo feminino. Em relação à idade, embora a tabela possa levar a pensar que a predominância foi do grupo de 20 a 49 anos $(48,44 \%)$, quando se calcula por ano de idade, a frequiência recai sobre a adolescência, ou seja, entre 10 e 19 anos, conforme Tabela III. Esses dados são coincidentes com os encontrados por outros autores ${ }^{(5,14)}$.

Ressalta-se a predominância de clientes atendidos na Unidade de Internação em Ortopedia no grupo etário de 20 a 49 anos em ambos os sexos devido à fase economicamente produtiva desta população e do grupo etário de 10 - 19 anos, devido a interferência na atividade escolar, lembrando o conseqüente absenteísmo ocasionado pela internação e pelo período necessário para a recuperação e reabilitação.

Dessa maneira, torna-se necessária a participação, na equipe multiprofissional, do assistente social na orientação dos recursos sociais, do fisioterapeuta, agilizando a reabilitação, prevenindo complicações decorrentes dos problemas ortopédicos, além do médico, enfermeira, nutricionista, psicólogo e terapeuta ocupacional.

Destacam-se, na Tabela IV, os diagnósticos predominantes nas diferentes faixas etárias e sexo. 


\begin{tabular}{|lrc|}
\hline $\begin{array}{l}\text { Tabela II - Distribuicão numérica e percentual das saídas hospitalares de } 1992 \text { a } \\
\text { Internação de Ortopedia HCRP, segundo os capítulos do CID (1995). Ribeirão Preto, 1999 }\end{array}$ & na Unidade de \\
\hline Capítulos do CID & № & $\%$ \\
\hline XVII Lesões e envenenamentos(800-999) & 2.345 & 40,30 \\
XIII Doenças do sistema osteomuscular e do tecido conjuntivo (710-739) & 1.904 & 32,72 \\
II Neoplasmas (140-239) & 479 & 8,23 \\
VI Doenças do sistema nervoso e dos órgãos dos sentidos (320-389) & 433 & 7,44 \\
XIV Anomalias congênitas(740-759) & 333 & 5,72 \\
V 01-82 & 143 & 2,46 \\
XII Doenças da pele e do tecido celular subcutâneo (680-709) & 49 & 0,85 \\
I Doenças infecciosas e parasitárias (001-139) & 42 & 0,72 \\
XVI Sintomas, sinais e afecções definidas(780-799) & 38 & 0,65 \\
III Doenças das glândulas endócrinas, nutrição, metabolismo e transtornos imunitários (240-279) & 19 & 0,33 \\
Demais CID & 17 & 0,29 \\
XV Algumas afecções originadas no período perinatal(760-769) & 14 & 0,24 \\
Não Válidos & 03 & 0,05 \\
\hline Total & 5.819 & 100,00 \\
\hline
\end{tabular}

\begin{tabular}{|c|c|c|c|c|c|c|}
\hline \multirow{2}{*}{$\begin{array}{c}\text { Faixa etária } \\
\text { (em anos }\end{array}$} & \multicolumn{2}{|c|}{ Masculino } & \multicolumn{2}{|c|}{ Feminino } & \multicolumn{2}{|c|}{ Total } \\
\hline & № & $\%$ & № & $\%$ & № & $\%$ \\
\hline$<1$ & 25 & 0,77 & 32 & 1,25 & 57 & 0,98 \\
\hline $1-4$ & 90 & 2,77 & 125 & 4,88 & 215 & 3,70 \\
\hline $5-9$ & 184 & 5,65 & 132 & 5,15 & 316 & 5,43 \\
\hline $10-14$ & 249 & 7,65 & 212 & 8,27 & 461 & 7,93 \\
\hline $15-19$ & 327 & 10,05 & 185 & 7,22 & 512 & 8,80 \\
\hline $20-49$ & 1.793 & 55,10 & 1.024 & 39,97 & 2.817 & 48,44 \\
\hline $50-69$ & 456 & 14,01 & 625 & 24,40 & 1.081 & 18,59 \\
\hline 70 ou + & 130 & 4,00 & 227 & 8,86 & 357 & 6,14 \\
\hline Total & 3.254 & 100,0 & 2.562 & 100,0 & $5.816^{*}$ & 100,00 \\
\hline
\end{tabular}

* Os três casos não válidos foram desconsiderados nesta tabela. 


\begin{tabular}{|c|c|c|c|c|c|c|}
\hline \multirow{2}{*}{$\begin{array}{l}\text { IDADE } \\
\text { (em anos) }\end{array}$} & \multicolumn{3}{|l|}{ MASCULINO } & \multicolumn{3}{|l|}{ FEMININO } \\
\hline & Diagnóstico & № & $\%$ & Diagnóstico & № & $\%$ \\
\hline \multirow[t]{3}{*}{$<1$} & $\begin{array}{l}\text { Deformidades Osteomusculares } \\
\text { Congênitas }\end{array}$ & 15 & 60,0 & $\begin{array}{l}\text { Deformidades Osteomusculares } \\
\text { Congênitas }\end{array}$ & 22 & 68,7 \\
\hline & Outros & 10 & 40,0 & Outros & 10 & 31,3 \\
\hline & Total & 25 & 100,0 & Total & 32 & 100,0 \\
\hline \multirow[t]{3}{*}{$1-4$} & $\begin{array}{l}\text { Fraturas de outras partes e partes não } \\
\text { especificadas do fêmur }\end{array}$ & 30 & 33,3 & $\begin{array}{l}\text { Deformidades Osteomusculares } \\
\text { Congênitas }\end{array}$ & 48 & 38,4 \\
\hline & Outros & 60 & 66,4 & Outros & 77 & 61,6 \\
\hline & Total & 90 & 100,0 & Total & 125 & 100,0 \\
\hline \multirow[t]{4}{*}{$5-9$} & Osteocondropatias & 29 & 15,8 & Deformidades Osteomusculares & & \\
\hline & $\begin{array}{l}\text { Fraturas de outras partes e partes não } \\
\text { especificadas do fêmur }\end{array}$ & 24 & 13,0 & $\begin{array}{l}\text { Congênitas } \\
\text { Osteocondropatias }\end{array}$ & $\begin{array}{l}20 \\
17\end{array}$ & $\begin{array}{l}15,1 \\
12,9\end{array}$ \\
\hline & Outros & 131 & 71,2 & Outros & 95 & 72,0 \\
\hline & Total & 184 & 100,0 & Total & 132 & 100,0 \\
\hline \multirow[t]{5}{*}{$10-14$} & \multirow{2}{*}{$\begin{array}{l}\text { Efeitos tardios de lesões traumáticas } \\
\text { osteomusculares e do tecido conjuntivo }\end{array}$} & & & Desvios da Coluna vertebral & 38 & 18,0 \\
\hline & & 33 & 13,3 & Osteocondropatias & 24 & 11,3 \\
\hline & Osteocondropatias & 28 & 11,2 & Outros & 150 & 70,7 \\
\hline & Outros & 188 & 75,5 & Total & 212 & 100,0 \\
\hline & Total & 249 & 100,0 & & & \\
\hline \multirow[t]{5}{*}{$15-19$} & \multirow{2}{*}{$\begin{array}{l}\text { Efeitos tardios de lesões traumáticas } \\
\text { osteomusculares e do tecido conjuntivo }\end{array}$} & & & Desvios da Coluna Vertebral & 35 & 18,9 \\
\hline & & 33 & 10,1 & Outros & 150 & 81,1 \\
\hline & Neoplasma maligno & 32 & 9,8 & Total & 185 & 100,0 \\
\hline & Outros & 262 & 80,1 & & & \\
\hline & Total & 327 & 100,0 & & & \\
\hline \multirow[t]{5}{*}{$20-49$} & $\begin{array}{l}\text { Efeitos tardios de lesões traumáticas } \\
\text { osteomusculares e do tecido conjuntivo }\end{array}$ & 193 & 10,8 & $\begin{array}{l}\text { Mononeurite do membro supe- } \\
\text { rior e Mononeurites múltiplas }\end{array}$ & 183 & 17,9 \\
\hline & \multirow{2}{*}{$\begin{array}{l}\text { Outros transtornos dos ossos e } \\
\text { cartilagens }\end{array}$} & & & Outros & 841 & 82,1 \\
\hline & & 154 & 8,6 & Total & 1024 & 100,0 \\
\hline & Outros & 1446 & 80,6 & & & \\
\hline & Total & 1793 & 100,0 & & & \\
\hline \multirow[t]{5}{*}{$50-69$} & Osteoartrose e transtornos afins & 40 & 8,8 & Mononeurite do membro supe- & & \\
\hline & \multirow{2}{*}{$\begin{array}{l}\text { Efeitos tardios de lesões traumáticas } \\
\text { osteomusculares e do tecido conjuntivo }\end{array}$} & & & & 89 & 14,2 \\
\hline & & 36 & 7,9 & Osteoartrose e transtornos afins & 88 & 14,1 \\
\hline & Outros & 380 & 83,3 & Outros & 448 & 71,7 \\
\hline & Total & 456 & 100,0 & Total & 625 & 100,0 \\
\hline \multirow[t]{4}{*}{70 ou +} & Fratura do colo do fêmur & 24 & 18,5 & Fratura do colo do fêmur & 75 & 33,0 \\
\hline & Outros & 106 & 81,5 & Osteoartrose & 25 & 11,0 \\
\hline & Total & 130 & 100,0 & Outros & 127 & 56,0 \\
\hline & & & & Total & 227 & 100,0 \\
\hline
\end{tabular}


Pode-se observar que, na faixa etária $<1$ ano, as Deformidades Osteomusculares Congênitas foram responsáveis pelo maior número de saídas em ambos os sexos, mantendo-se, nas faixas etárias de 1 a 4 e de 5 a 9 anos, para o sexo feminino e, diferindo para o sexo masculino, em que as Fraturas de Outras Partes não Específicas do Fêmur e as Osteocondropatias foram predominantes.

Nas faixas etárias de 10 a 14 e de 15 a 19 anos, a predominância recaiu sobre os Desvios da Coluna Vertebral para o sexo feminino, e, para o sexo masculino, Os Efeitos Tardios das Lesões Traumáticas Osteomusculares e do Tecido Conjuntivo. Este mesmo diagnóstico foi predominante para o sexo masculino das faixas etárias de 20 a 49 e de 50 a 69 anos e, para o sexo feminino, as Mononeurites de Membro Superior e Mononeurite Múltipla.

As fraturas do colo do fêmur prevaleceram em ambos os sexos da faixa etária de 70 anos ou + .

Na Tabela IV, pode-se notar a predominância do mesmo diagnóstico em diferentes faixas etárias, relevando os efeitos tardios de lesões traumáticas osteomusculares e do tecido conjuntivo. Considerase importante ressaltar que o tratamento da maioria dos diagnósticos citados requer tecnologia de ponta e interdisciplinariedade para intervenção eficaz, exigindo, em muitos casos, repetidas internações de longo período, além de acompanhantes, tendo em vista a tenra idade, o estado emocional dos clientes e o ancião. Diante disso, destaca-se a importância, dentro da estrutura física da Unidade de Internação de Ortopedia, de um espaço lúdico, destinado aos pacientes internados na unidade, e de um espaço destinado ao conforto e higiene das mães e acompanhantes.

\section{CONSIDERAÇÕES FINAIS}

É sabido que as informações a respeito do cliente, quando na saída hospitalar, são importantes na orientação do uso dos recursos dos serviços de saúde. Acredita-se que o conhecimento da morbidade hospitalar, da Unidade de Internação em Ortopedia, pelos enfermeiros, possa servir como subsídio para o planejamento e a avaliação da assistência de enfermagem prestada nesta unidade de internação, bem como para a otimização dos recursos humanos, materiais e físicos, utilizados, de modo a garantir a qualidade da assistência, quando se faz a internação.

Ressalta-se a predominância do Capítulo XVII - Lesões e Envenenamentos - (40,00\%), na ocorrência de pacientes traumatizados, o que exige a formação de recursos humanos especializados para o atendimento a tal clientela. Em nossa instituição, neste sentido, acredita-se que devam ser estabelecidas diretrizes, baseadas na morbidade prevalente, fundamentando os programas de educação continuada em enfermagem e a elaboração de protocolos de assistência.

MELO MRAC; FERRAZ CA; SOUZA CR \& LAVRADOR MAS. Morbidity study of the orthopedic unit. Medicina, Ribeirão Preto, 33: 73-81, jan./march 2000.

ABSTRACT: Study Design: Retrospective and descriptive research. Objective: the purpose of this study is to describe the clientele hospitalized at the Orthopedic Unit of the University of São Paulo at Ribeirão Preto Faculty of Medicine Hospital, according to variables such as the main diagnosis, age and sex. Method: Authors analyzed hospital discharge codified by the International Classification of Diseases, $9^{\text {th }}$ review, 1975, occurred from 1992 to 1996 . Data were collected through the Service of Medical Files of the Institution, through a program of registries of discharges from Data Process Company of São Paulo (PRODESP) and coded from a special software developed ein C++. Results: The authors found 5819 hospital discharge and the Chapter XVII Lesions and poisoning was the responsible for the highest number of hospital discharge, corresponding to 2.345 (40.30\%). Following, the Chapter XIII - Diseases of the osteomuscular and conjunctive tissue was responsible for 1.904 hospital discharge (32.72\%). The Chapter VI - Diseases of the nervous system was the responsible for 433 hospital discharge $(7.44 \%)$. In specific, the diagnostic of Carpal tunnel syndrome represented 310 cases. The Chapter II - Neoplasm was responsible for 479 hospital discharge (8.23). Chapter XIV - Congenital anomalies was responsible for 333 hospital discharge (5.72\%). The other chapters presented a lower frequency. In all chapters, authors found a distribution of several age groups, mainly from 20 to 49 years of age (48.44\%). Final Considerations: They believe that knowledge on hospital morbidity will orient the use of health services resources, in order to garantee the quality of care.

UNITERMS: Morbidity. Orthopedic Nursing. Hospital Units .Orthopedics. 


\section{REFERÊNCIAS BIBLIOGRÁFICAS}

1 - MELO MRAC. O Sistema Único de Saúde e as ações do enfermeiro da instituição hospitalar. Tese de Doutorado, Escola de Enfermagem de Ribeirão Preto da USP, Ribeirão Preto, p. 1152,1996 .

2 - REICHENHEIM ME \& WERNECK GL. Adoecer e morrer no Brasil dos anos 80: perspectivas de novas abordagens. In: GUIMARÃES R \& TAVARES RAW, org. Saúde e sociedade no Brasil: anos 80. Relume Dumará, Rio de Janeiro, p. 113-130, 1994.

3 - CASTELAR RM; IWERSEN M. O atual quadro sanitário do Brasil. In: CASTELAR RM; MORDELET P \& GRABOIS V, dir. Gestão hospitalar: um desafio para o hospital brasileiro. ENSP, Paris, p. 29-37, 1993.

4 - XAVIER CAM \& CARVALHEIRO JR. Incidência de fraturas, exceto de crânio, no município de Ribeirão Preto, SP nos anos de 1969 - 1970. Rev Saúde Pública, São Paulo 12: 432-442, 1978.

5 - LEBRÃO ML; LITVOC J; FIGUEIREDO GM \& LEITE RM. Estudo da morbidade dos pacientes internados no Hospital das Clínicas da Faculdade de Medicina da USP - 1989. Rev Hosp Clín Fac Med São Paulo. 48: 189-198, 1993.

6 - POLIT DF \& HUNGLER BP. Fundamentos de pesquisa em enfermagem. 3ª edição, Artes Médicas, Porto Alegre, 1995.

7 - TRIVIÑOS ANS. Introdução à pesquisa em ciências sociais: a pesquisa qualitativa em educação. Atlas, São Paulo, 1990.

8 - MANUAL DE CLASSIFICAÇÃO INTERNACIONAL DE DOENÇAS, LESÕES E CAUSAS DE ÓBITOS, 9aㅡ Revisão, 1975. Centro da OMS para Classificação de Doenças. Ministério da Saúde, Universidade de São Paulo, Organização Panamericana de Saúde, São Paulo, 1980.2v.
9 - FÁVERO M. Estudo epidemiológico dos acidentes de trânsito em Ribeirão Preto. Tese de Doutorado, Faculdade de Medicina de Ribeirão Preto - USP, Ribeirão Preto. p.1-135, 1971.

10 - KOIZUMI MS. Acidentes de motocicletas no município de São Paulo/SP, Brasil-1. Caracterização de acidente e da vítima. Rev Saúde Pública, São Paulo 19: 175-489, 1985.

11 - KOIZUMI MS. Padrão das lesões das vítimas de acidentes de motocicletas. Rev Saúde Pública, São Paulo 26: 305-315, 1992.

12 - BARROS MBA. Estudo da morbidade hospitalar no município de Ribeirão Preto em 1975. Dissertação de Mestrado, Faculdade de Medicina de Ribeirão Preto da USP, Ribeirão Preto, p. 1-109, 1975.

13 - MINAYO MCS. A violência social sob a perspectiva da saúde pública. Cad Saúde Pública, Rio de Janeiro 10:7-18, 1994. Supl.1

14-RODRIGUES EML. Morbidade hospitalar por lesões e envenenamentos em procedentes do município de Ribeirão Preto. Tese de Doutorado, Faculdade de Medicina de Ribeirão Preto da USP, Ribeirão Preto. p. 1-88, 1995.

15 - ANDRADE JI. Formação de recursos humanos para assistência ao traumatizado. Medicina, Ribeirão Preto 23: 269-270, 1990.

Recebido para publicação em 28/12/1999

Aprovado para publicação em 03/03/2000 